\title{
A New Closed Set in Topological Spaces
}

\author{
A. A. Azzam ${ }^{1,2}$ \\ ${ }^{1}$ Department of Mathematics, Prince Sattam Bin Abdulaziz University, Alkharj 11942, Saudi Arabia \\ ${ }^{2}$ Department of Mathematics, New Valley University, Elkharga 72511, Egypt
}

Correspondence should be addressed to A. A. Azzam; azzam0911@yahoo.com

Received 2 January 2021; Accepted 21 May 2021; Published 1 June 2021

Academic Editor: Francisco Rossomando

Copyright $\odot 2021$ A. A. Azzam. This is an open access article distributed under the Creative Commons Attribution License, which permits unrestricted use, distribution, and reproduction in any medium, provided the original work is properly cited.

Our purpose of this work is to implement a class of $s \hat{g}$-closed sets, which is property placed among the classes of semiclosed sets and $g s$-closed sets. The relations with other concepts directly or indirectly joined with generalized closed sets are inspected. In addition, as an application, using the notion of $s \hat{g}$-closed sets, we give a brief expansion of a new space named $T_{\hat{s} \hat{g}}$-space.

\section{Introduction and Preliminaries}

Topological space has become one of the most important spaces that help to solve many of the contemporary problems. It is the reference for measuring the descriptive rather than quantitative. Also, closed sets are essential matters in a space that carries topology. For instance, one can know the topology on a set by using either the axioms for the closed sets or the Kuratowski closure axioms. In 1970, Levine [1] initiated the study of so-called generalized closed sets. By definition, a subset $B$ of the space $Y$ which carries topology $\tau$ is named generalized closed set if the closure of any subset $B$ of $Y$ is included in each open superset of $B$. This notion has been studied extensively in recent years by many topologists because generalized closed sets are not only natural generalizations of closed sets. Furthermore, the study of generalized closed sets also extends new depictions of some known classes of spaces, for instance, the class of extremely disconnected spaces. In 1987, Bhattacharyya and Lahiri [2] introduced a new class of sets named semigeneralized closed sets using semiopen sets of Levine [3] which obtained various properties corresponding to [1]. In 1990, Arya and Nour [4] defined the generalized semiclosed sets. Dontchev [5] has introduced $g s p$-closed sets by generalizing semipreopen sets. Generalized closed sets in bitopological spaces have been introduced by Fukutake [6]. More recently, Kumar [7] introduced $\hat{g}$-closed sets in topological spaces. John and Sundaram [8] introduced and studied the concept of $g^{*}$-closed sets and $T_{1 / 2}^{*}$-space concerning bitopological space. Noiri and Rajesh [9] introduced generalized closed sets with respect to an ideal in bitopological spaces. Noiri and popa [10] studied the relation between $*$-closed sets and $I$ - $g$-closed sets in ideal topological spaces. In 2018, AlSaadi [11] discussed the concept of strongly $g^{*}$-closed sets and strongly $T_{1 / 2}^{*}$-spaces in bitopological spaces. In the present work, we introduce the notion of $s \hat{g}$-closed sets and find some basic properties for it. We are also showing that this class lies among the class of semiclosed sets and the class of $g s$ - closed sets. Applying the sets, we introduce a new space called $T_{s \hat{g}}$-space. Let us review some of the standard facts on near closed sets as the next.

Definition 1. A subset $B$ of a space $Y$ which carries topology $\tau$ is named as follows:

(i) Semiclosed [12] if $\operatorname{Int}(C l(B)) \subseteq B$,

(ii) Semiopen [3] if $Y \backslash B$ is semiclosed or equivalently, if $B \subseteq C l(\operatorname{Int}(B))$,

(iii) Semi-preclosed [13] if $\operatorname{Int}(C l(\operatorname{Int}(B))) \subseteq B$,

(iv) Semi-preopen [13] if $Y \backslash B$ is semi-preclosed or equivalently, if $B \subseteq C l(\operatorname{Int}(C l(B)))$.

(v) Generalized closed [1] if $C l(B) \subseteq Y$ whenever $B \subseteq U$ and $U$ is open set in $(Y, \tau)$. The semiclosure $[12,14]$ of a subset $B$ of $(Y, \tau)$, denoted by $s C l_{Y}(B)$, briefly $s C l(B)$, is defined to the intersection of all semiclosed sets containing $B$. The semi-interior of $B$ [12], denoted by $\operatorname{sint}(B)$, is defined by the union of all 
semiopen sets contained in B. A number of definitions and depictions have been handled in $[1,2,4,5,7]$ with respect to generalized closed sets or $T_{\hat{g}}$-space.

\section{Basic Properties of $s \widehat{g}$-Closed Sets}

In section 2 , we give a brief exposition of $s \hat{g}$-closed sets, some of their properties, and relations with other known classes of subsets.

Definition 2. Let $Y$ be a nonempty set that carries topology $\tau$; a subset $B$ of $Y$ is called $s \hat{g}$-closed if $s C l(B) \subseteq G$ whenever $B \subseteq G$ and $G$ is a $\hat{g}$-open set in $(Y, \tau)$.

Theorem 1. Let $Y$ be a nonempty set that carries topology $\tau$. Then, the next declaration is verified.

(i) Each semiclosed set is s $\widehat{g}$-closed in $(Y, \tau)$

(ii) Each $g$-closed set is s $\hat{g}$-closed in $(Y, \tau)$

(iii) Each $s \hat{g}$-closed set is $g s$-closed and hence gsp-closed in $(Y, \tau)$

(iv) Each $\alpha \widehat{g}$-closed set is s $\widehat{g}$-closed in $(Y, \tau)$

Proof

(i) Let $B$ be a semiclosed set and $G$ be any $\widehat{g}$-open containing $B$. Since $B$ is semiclosed, $s C l(B)=B$ for each subset $B$ of $Y$. Therefore, $s C l(B) \subseteq G$, and hence $B$ is $s \hat{g}$-closed.

(ii) It is obvious from inclusion $s \mathrm{Cl}(B) \subset \mathrm{Cl}(B)$ for each subset $B$ of $(Y, \tau)$.

(iii) It is clear that $s p C l(B) \subseteq s C l(B)$ for each subset $B$ of $(Y, \tau)$, and each open set is $\widehat{g}$-open.

(iv) From $s C l(B) \subseteq \alpha C l(B)$ for each subset $B$ of $(Y, \tau)$, the proving is clear.

Remark 1. Figure 1 shows the connections results among $s \widehat{g}$-closed and different types of other sets.

In Remark 1, the relationships cannot be reversible as the next instance shown.

Example 1. Let $Y=\{1,2,3\}$ carry topology $\tau$.

(i) If $\tau=\{\phi,\{1,2\}, Y\}$, then $\{1,3\}$ is $s \hat{g}$-closed but neither semiclosed nor $* g$-closed.

(ii) If $\tau=\{\phi,\{1,3\},\{1\},\{3\}, Y\}$, then $\{1\}$ is $g s$-closed and gsp closed but not $s \widehat{g}$-closed.

(iii) If $\tau=\{\phi,\{1,3\},\{1\}, Y\}$, then $\{3\}$ is $s \hat{g}$-closed but not $* g$-closed. Also, $\{1,2\}$ is $s \hat{g}$-closed but not semiclosed.

Remark 2. The following examples from (i) to (iii) show that the concept of $s \widehat{g}$-closedness is independent from $\widehat{g}$-closedness, $g$ closedness, $s g$-closedness, and spg-closedness. (i) Let $Y=\{1,2,3\}$ and $\tau=\{\phi,\{1\},\{1,3\}, Y\}$. In a space $(Y, \tau)$, a subset $\{1,2\}$ is $s \hat{g}$-closed but it is not $s p g$-closed. Also, a subset $\{3\}$ is $s \hat{g}$-closed but neither $g$-closed nor $\widehat{g}$-closed in $(Y, \tau)$.

(ii) Let $(Y, \tau)$ be the same space in Example 1(ii). A subset $\{2\}$ is semiclosed and $s g$-closed but it is not $s \widehat{g}$-closed.

(iii) Let $\tau$ be the usual topology on the real line $\mathbb{R}$. One can deduce that the open interval $(1,2)$ is $s g$-closed but not $s \hat{g}$-closed.

Theorem 2. Let $B$ and $C$ be two s $\widehat{g}$-closed subsets of $(Y, \tau)$. Then,

(i) The union of two s $\hat{g}$-closed sets is also s $\hat{g}$-closed in $(Y, \tau)$

(ii) If $B$ is $\widehat{g}$-open and $s \hat{g}$-closed, then $B$ is semiclosed in $(Y, \tau)$

(iii) If $B$ is $s \hat{g}$-closed in $(Y, \tau)$, then $s C l(B) / B$ does not contain any nonempty $\widehat{g}$-closed set

(iv) If $B$ is s $\widehat{g}$-closed of $(Y, \tau)$ such that $B \subseteq C \subseteq s C l(B)$, then $C$ is also $s \hat{g}$-closed of $(Y, \tau)$

(v) For each $x \in Y,\{x\}$ is $\widehat{g}$-closed or $X \backslash\{x\}$ is s $\widehat{g}$-closed of $(Y, \tau)$

(vi) Every subset is $s \hat{g}$-closed of $(Y, \tau)$ if and only if every $\widehat{g}$-open set is semiclosed

Proof

(i) Let $B$ and $C$ be two $s \hat{g}$-closed sets and $U$ be any $\hat{g}$-open set such that $B \cup C \subseteq U$. Then, $s C l(B \cup C)=s C l(B) \cup s C l(C) \subseteq U$.

(ii) Since $B$ is $\widehat{g}$-open and $s \widehat{g}$-closed, $s C l(B) \subseteq B$. Hence, $B$ is semiclosed.

(iii) Assume that there exists a $\hat{g}$-closed set $F$ such that $F \subseteq s C l(B) / B$. Then, $B \subseteq X \backslash s C l(B)$ and so $s C l(B) \subseteq Y \backslash F$. Hence, we have that $F \subseteq Y \backslash s C l(B)$ and $F=\phi$ because $F \subseteq s C l(B)$.

(iv) Let $U$ be a $\widehat{g}$-open set such that $C \subseteq U$. Then, we have that $s C l(B) \subseteq U$ and $C l(C) \subseteq s C l(B) \subseteq U$. Therefore, $C$ is $s \hat{g}$-closed in $(Y, \tau)$.

(v) If $\{x\}$ is not $\hat{g}$-closed, then $Y \backslash\{x\}$ is not $\hat{g}$-open. Therefore, $X \backslash\{x\}$ is $s \hat{g}$-closed in $(Y, \tau)$.

(vi) Let $U$ be a $\hat{g}$-open set. Then, we have that $s C l(U) \subseteq U$ and hence $U$ is semiclosed. Conversely, let $B$ be a subset and $U$ a $\hat{g}$-open set such that $B \subseteq U$. Then, $s C l(B) \subseteq s C l(U)=U$, and hence $B$ is $s \hat{g}$-closed.

Theorem 3. A subset $B$ of $(Y, \tau)$ space is $s \hat{g}$-closed if and only if $\operatorname{sCl}(A) / B$ contains no nonempty $\hat{g}$-closed set in $(Y, \tau)$.

Proof. Let $F$ be a nonempty $\hat{g}$-closed set of $(Y, \tau)$ such that $F \subseteq s C l(B) \backslash B$. Since $Y \backslash F$ is $\hat{g}$-open and $B$ is $s \widehat{g}$-closed, $\quad s C l(B) \subseteq X \backslash F, \quad$ i.e., $\quad F \subseteq Y \backslash s C l(B)$. Then, $F \subseteq$ 


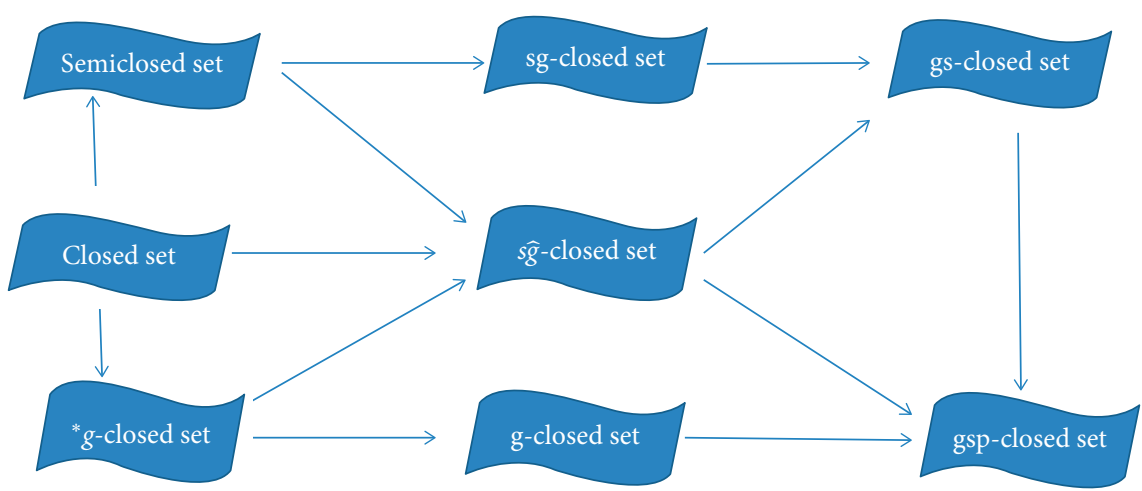

FIGURE 1: Relationships between some classes of closed sets.

$s C l(B) \cap(Y \backslash s C l(B))=\phi$ and hence $F=\phi$, which is a contradiction.

Conversely, let $G$ be a $\hat{g}$-open set such that $A \subseteq G$. Suppose that $s C l(B)$ is not contained in $G$. Then, by Remark $1, \quad s C l(B) \cap(X, G)$ is nonempty $\hat{g}$-closed and $s C l(B) \cap(Y, G) \subseteq s C l(B) \backslash B$, which is a contradiction.

Remark 3. The intersection of two $s \hat{g}$-closed sets need not be $s \widehat{g}$-closed as the following instance shows.

Example 2. Let $Y=\{1,2,3,4\}$ with a topology $\tau=\{\phi,\{1\}$, $\{2\},\{1,2\},\{1,2,3\}, Y\}$. Put $B=\{1,2,4\}$ and $C=\{1,3,4\}$. Then, $B$ and $C$ are $s \hat{g}$-closed but $B \cap C=\{1,4\}$ is not $s \widehat{g}$-closed in $(Y, \tau)$.

\section{3. $s \widehat{g}$-Open Sets}

In the third section, we explain the definition of $s \hat{g}$-open and related by $s \hat{g}$-closed with some examples.

Definition 3. A subset $B$ of a space $Y$ which carries topology $\tau$ is named $s \hat{g}$-open if $Y \backslash B$ is $s \widehat{g}$-closed.

Remark 4. For A subset $B$ of a space $Y$ which carries topology $\tau, s C l(Y \backslash B)=Y \backslash \operatorname{sint}(B)$.

Theorem 4. A subset $B$ is $s \hat{g}$-open in $(Y, \tau)$ if and only if $F \subseteq \operatorname{sint}(B)$, whenever $F \subseteq B$ and $F$ is $\hat{g}$-closed in $(\underline{Y}, \tau)$.

Proof. Let $F$ be a $\widehat{g}$-closed of $(Y, \tau)$ and $F \subseteq B$. Then, $Y \backslash F$ is $\widehat{g}$-open and $Y \backslash B \subseteq Y \backslash F$. Since $Y \backslash B$ is $s \hat{g}$-closed, $s C l(Y \backslash B) \subseteq Y \backslash F$, that is, $Y \backslash s \operatorname{Int}(B) \subseteq Y \backslash F$. Hence, $F \subseteq \operatorname{sint}(B)$.

Conversely, let $G$ be a $\hat{g}$-open set of $(Y, \tau)$ and $Y \backslash B \subseteq G$. Since $Y \backslash G$ is a $\hat{g}$-closed set contained in $A$, by hypothesis

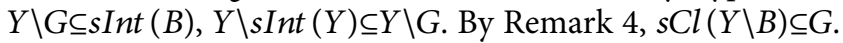
Hence, $Y \backslash A$ is $s \hat{g}$-closed, and so $B$ is $s \hat{g}$-open.

Theorem 5. A subset $B$ is $s \hat{g}$-closed in $(Y, \tau)$ if and only if $s C l(B) \backslash B$ is s $\widehat{g}$-open.

Proof. Let $F$ be a $\hat{g}$-closed set of $(Y, \tau)$ such that $F \subseteq s C l(B) \backslash B$. Since $B$ is $s \widehat{g}$-closed, by Theorem $4, F=\phi$ that $F \subset \operatorname{sint}(s C l(B)) \backslash B$ and $s C l(B) \backslash B$ is $s \hat{g}$-open.
Reciprocally, let $B \subseteq G$, we have $G$ as $\widehat{g}$-open in $(Y, \tau)$. Now, $\quad s C l(B) \cap(Y, G) \subset s C l(B) \cap(Y, B)=s C l(B) \backslash B$ and since $s C l(B) \cap(Y, G)$ is $\hat{g}$-closed and $s C l(B) B$ is $s \hat{g}$-open, $s C l(B) \cap(Y, G) \subset \operatorname{sint}(s C l(B)) \backslash B=\phi$. Then, $s C l(B) \cap$ $(Y, G)=\phi$ or $s C l(B) \subseteq G$, and hence $B$ is $s \hat{g}$-closed.

Example 3. Let $\{=\{1,2,3\}$ with topology $\tau=\{\phi, Y,\{1\}$, $\{1,2\}$. In $(Y, \tau)$, the set $\{2\}$ is $s \widehat{g}$-open.

As an application of the concept of $s \hat{g}$-closed sets, we introduce the following space called $T_{\hat{s g}}$-space.

Definition 4. A space $Y$ which carries topology $\tau$ is called a $T_{\widehat{s} \hat{g}}$-space if each $s \hat{g}$-closed set is closed in $Y$.

Remark 5. The spaces $T_{\widehat{s g}}$ and $T_{\widehat{g}}$ are independent as seen from the next instances.

Example 4. Let $Y=\{1,2,3\}$ with topology $\tau=\{\phi, Y,\{1\}\}$. Then, the space $(Y, \tau)$ is $T_{\hat{g}}$-space but not a $T_{s \hat{g}}$-space.

Example 5. Let $Y=\{1,2,3\}$ with $\tau=\{\phi, Y,\{1\},\{2,3\}\}$. Then, $(Y, \tau)$ is $T_{\hat{s g}}$-space but not $T_{\hat{g}}$-space.

\section{Conclusion}

Popularizations of closed sets in point-set topology will give some new topological properties (for instance, separation axioms, compactness, connectedness, and continuity) and a brief expansion of a new space named $T_{s \hat{g}^{-}}$-space which have been found to be very beneficial in the study of certain objects of digital topology. Thus, we may stress once more the importance of $s \hat{g}$-closed sets as a branch of them and the possible application in computer [15-17] and quantum.

\section{Data Availability}

The data are included only in references of manuscript.

\section{Conflicts of Interest}

The author declares that there are no conflicts of interest. 


\section{References}

[1] N. Levine, "Generalized closed sets in topology," Rendiconti del Circolo Matematico di Palermo, vol. 19, pp. 89-96, 1970.

[2] P. Bhattacharyya and B. K. Lahiri, "Semi-generalized closed sets in toplogy," Indian Journal of Mathematics, vol. 29, pp. $375-382,1987$.

[3] N. Levine, "Semi-open sets and semi-continuity in topological spaces," The American Mathematical Monthly, vol. 70, no. 1, pp. 36-41, 1963.

[4] S. P. Arya and T. Nour, "Charaterizations of s-normal space," Indian Journal of Pure and Applied Mathematics, vol. 21, pp. 717-719, 1990.

[5] J. Dontchev, "On generaizing semi-preopen sets," Mem. Fac. Sci. Kochi Univ. Ser.A Math, vol. 16, pp. 35-48, 1995.

[6] T. Fukutake, "On generalized closed sets in bitopological spaces," Bulletin of Fukuoka University of Education, vol. 35, pp. 19-28, 1985.

[7] V. Kumar, "g-closed sets in topological spaces," The Allahabad Mathematical Society, vol. 18, pp. 99-122, 2003.

[8] M. S. John and P. Sundaram, " $g$ *-closed sets in bitopological spaces," Indian Journal of Pure and Applied Mathematics, vol. 35, no. 1, pp. 71-80, 2004.

[9] T. Noiri and N. Rajesh, "Generalized closed setswith respect to an ideal bitopological spaces," Acta Mathematica Hungarica, vol. 125, 2009.

[10] T. Noiri and V. popa, "Between *-closed sets and $I$-g-closed sets in ideal topological spaces," Rendiconti del Circolo Matematico di Palermo, vol. 59, no. 2, pp. 251-260, 2010.

[11] H. Al-Saadi, "Strongly $g^{*}$-closed sets and strongly $T_{1 / 2}^{*}$ spaces in bitopological spaces," Hacettepe Journal of Mathematics and Statistics, vol. 47, no. 1, pp. 37-45, 2018.

[12] S. G. Crossely and S. K. Hildebrand, "Semi-closure," Texas Journal of Science, vol. 22, pp. 99-112, 1971.

[13] D. Andrijevic, "Semi-preopen sets," Matematički Vesnik, vol. 38, no. 1, pp. 24-32, 1986.

[14] N. Biswas, "On characterizations of semi-continuous functions," Atti della Accademia Nazionale dei Lincei. Classe di Scienze Fisiche, Matematiche e Naturali, vol. 8, pp. 399-402, 1970.

[15] M. S. El Naschie, "On the uncertainty of Cantorian geometry and two, slit Experiment, Chaos," Chaos, Solitons \& Fractals, vol. 9, no. 3, pp. 517-529, 1998.

[16] E. D. Kalimsky, R. Kopperman, and P. R. Meyer, "Computer graphics and connected topologies on finite ordered sets," Topology and its Applications, vol. 36, pp. 1-17, 1990.

[17] T. Y. Kony, R. Kopperman, and P. R. Meyer, "A topological approach to digital toplogy," The American Mathematical Monthly, vol. 98, pp. 901-917, 1991. 